\title{
CREATING A PROGNOSTIC MODEL TO ASSESS THE MULTIVARIATE PREDICTORS OF NECK METASTASIS IN ORAL CAVITY SQUAMOUS CELL CARCINOMA.
}

\author{
S. Meghji, L. Langstaff, I. Nunney And R. Nassif
}

\section{INTRODUCTION}

- H\&N cancer $8^{\text {th }}$ most common cancer. Oral cancer is predicted to rise by $33 \%$ by 2035 . Majority of malignant tumours $=$ oral cavity squamous cell carcinoma (OCSCC).

- UK Head and Neck Guidelines have suggested that surgery remains the main management strategy for primary OCSCC. The management of clinically node negative neck OCSCC has remained the topic of much debate over the last 30 years. Some OCSCC have a high risk of occult metastasis even if the patient is clinically node negative.

- Tumour thickness and depth of invasion = risk factors for nodal metastasis. Critical tumour depth has ranged from $4 \mathrm{~mm}$ to $10 \mathrm{~mm}$. Depth of invasion has just been added to the TNM staging. Angiolymphatic spread, perineural invasion, increasing T-stage and poorly differentiated cancer have all been found to predict nodal disease.

\section{HYPOTHESIS}

- Can the combined characteristics of the primary oral cavity tumour predict nodal disease?

\section{METHODOLOGY}

- Retrospective case note review in a large teaching hospital between June 2011 - March 2016 of all patients treated surgically for an OCSCC and who had a neck dissection. Data collected included: Location of the primary cancer, tumour thickness, tumour diameter, angiolymphatic spread, perineural invasion, tumour differentiation, lymph node status and TNM stage.

\section{RESULTS}

- 168 patients underwent primary tumour excision with (195) selective neck dissections (unilateral or bilateral) over a five year period . 91 males, 77 females. The tumor subsite: Tongue - 69 patients, Mandible - 30 patients, Floor of mouth - 29 patients, Retromolar - 17 patients, Maxilla - 14 patients and Buccal - 9 patients.

- All patients were followed up for at least 1 year. $62 \%$ of neck did not yield a positive lymph node, $38 \%$ did yield one or more positive lymph nodes.

- Univariate analysis showed a statistical significant association of tumour thickness depth $(\mathrm{P}=0.0007)$, tumour diameter $(\mathrm{P}=0.0005), \quad$ angiolymphatic $(\mathrm{P}<0.0001)$, perineural spread $(\mathrm{P}=0.0127)$, tumour location $(\mathrm{P}=0.0025)$ and tumour differentiation $(\mathrm{P}=0.0015)$ with having a positive cervical lymph node.
- A Nomogram was formulated using a logistic regression model with forward stepwise model selection. It included tumour location, tumour depth and angiolymphatic spread, The logistic model had a concordance of $81 \%$, a sensitivity of $70.5 \%$ and specificity of $70.7 \%$.

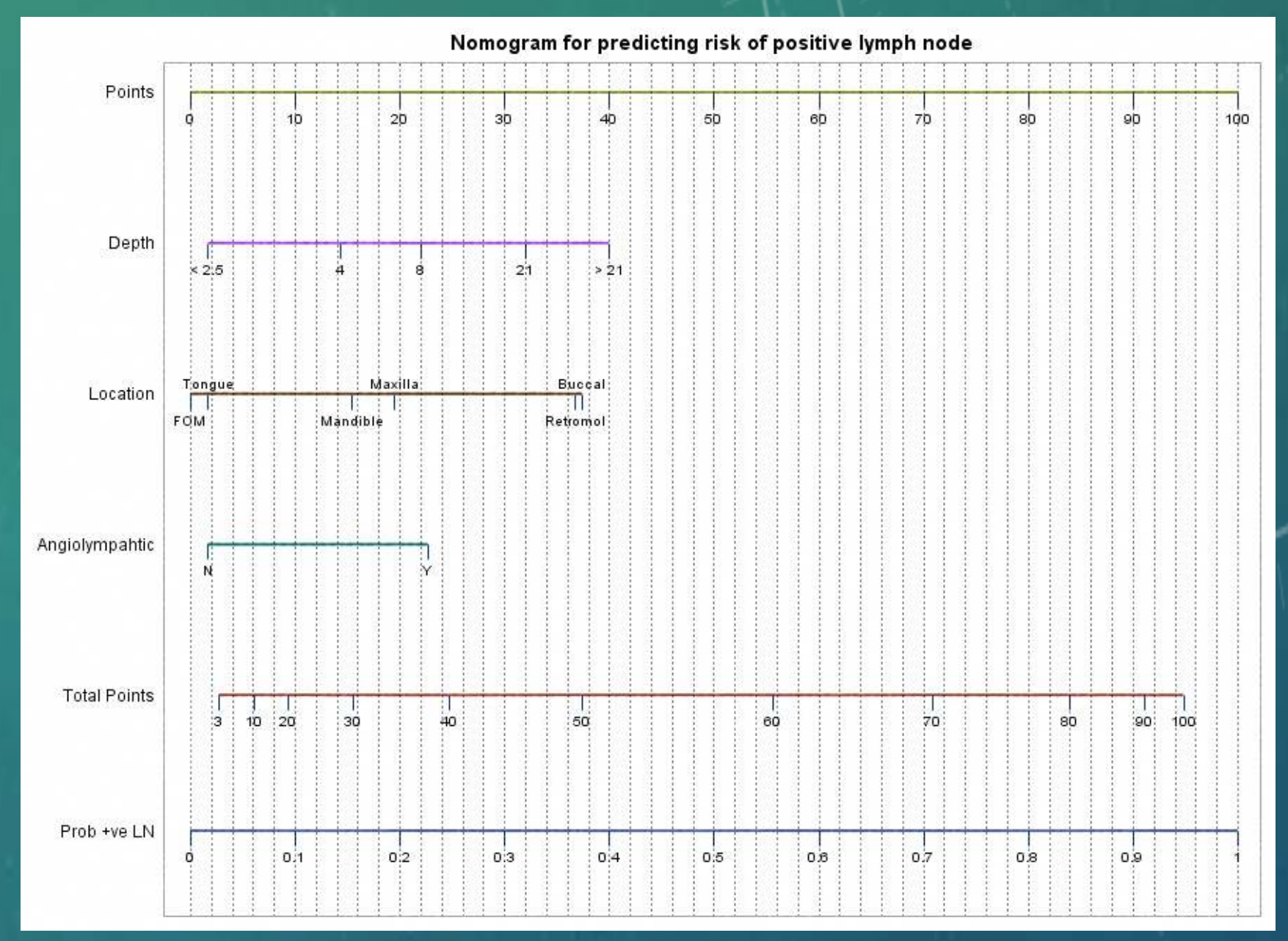

DISCUSSION

- Our research agrees with other studies that tumour thickness is an important characteristic in predicting cervical lymph node metastasis. It has also highlighted that the other characteristics of the primary tumour; perineural invasion, angiolymphatic spread and tumour differentiation also help to predict nodal disease. We created a nomogram model to try and predict the risk of lymph node metastasis by combining the characteristics of the primary tumour.

- Our limitations are that it was a retrospective study. Small number of patients in each oral subsite, it was difficult to extract what the initial clinical T-stage was for these patients. We only sampled patients with OCSCC who had a neck dissection. Clinical application is still uncertain and external validation of the model is required.

\section{CONCLUSION}

- To our knowledge this is the first nomagram model to be used to try and predict the risk of lymph node metastasis from the characteristics of the primary cancer. It is a pictorial model, which is easy to use and understand. If the variables in the model can be illustrated in the FNA or core biopsy along with imaging then it can potentially be used to provide probability of neck disease prior to primary surgery. This is a prototype for a bigger project to create an OCSCC surgical risk calculator to predict nodal disease. 